IUHET 302

IU/NTC 95-06

May 26, 1995

\title{
New formulas relating the masses of some baryons and mesons
}

\author{
D. B. Lichtenberg and R. Roncaglia \\ Physics Department, Indiana University, Bloomington, IN 47405, USA
}

Sum rules relating the masses of ground-state baryons and mesons are obtained in a constituent quark model. The interaction is assumed to be independent of quark spins except for a spindependent part that can be treated as a perturbation. Where data are available, the sum rules agree with experiment to better than $1 \%$.

PACS numbers: 12.10.Kt, 12.40.Yx

In this work we consider meson and baryon masses using a Hamiltonian formalism within the framework of the constituent quark model. We do not assume any particular functional form for the spin-independent part of the Hamiltonian. However, we treat the spin-dependent part as a perturbation, and we confine ourselves to ground-state hadrons, so that the spin dependence arises from the spin-spin or colormagnetic term. With these modest inputs plus an additional approximation (to be discussed later), we are able to obtain three sum rules relating the masses of certain baryons and mesons. Two of these sum rules agree with experiment to better than $1 \%$ and the third remains to be tested.

In the constituent quark model, the mass of a hadron can be written as the sum of the masses of its constituent quarks plus an interaction energy. The mass $M_{M}(i j)$ of a meson composed of a quark $q_{i}$ and an antiquark $\bar{q}_{j}$ with masses $m_{i}$ and $m_{j}$ respectively is given by

$$
M_{M}(i j)=m_{i}+m_{j}+E(i j),
$$

where $E(i j)$ is the interaction energy. Similarly, the mass $M_{B}(i j k)$ of a baryon containing quarks with masses $m_{i}, m_{j}$, and $m_{k}$ is

$$
M_{B}(i j k)=m_{i}+m_{j}+m_{k}+E(i j k),
$$

where $E(i j k)$ is the interaction energy. We assume that the interaction energies $E(i j)$ and $E(i j k)$, and consequently the masses $M_{M}(i j)$ and $M_{B}(i j k)$ are calculable in principle from a Hamiltonian without spin-dependent forces. We also assume that the mass of a constituent quark can be assigned a unique value, independent of the hadron in which it is bound, but we do not have to specify the quark masses to obtain our results.

It follows from Eqs. (1) and (2) that

$$
M_{B}(123)-M_{B}(453)-M_{M}(12)+M_{M}(45)=\delta(12345),
$$

where $\delta(12345)$ is defined by

$$
\delta(12345)=E(123)-E(453)-E(12)+E(45) .
$$


We immediately see from Eq. (3) that if $\delta(12345)$ is zero, we have the sum rule

$$
M_{B}(123)-M_{M}(12)=M_{B}(453)-M_{M}(45) .
$$

We next come to the important question of the circumstances under which Eq. (5) is most likely to hold to a good approximation. It has been previously found $[1,2]$ that the ground-state vector meson and spin $3 / 2$ baryon interaction energies are smooth, monotonically decreasing functions of the generalized reduced mass $\mu(12 \ldots)$ of the system, where $\mu(12 \ldots)$ is defined by

$$
1 / \mu(12 \ldots)=\sum_{i} 1 / m_{i}
$$

We have looked at the energies $E(i j)$ and $E(i j k)$ appropriately averaged over the spins of groundstate hadrons of different spins and the same quark content. We have verified numerically that, for reasonable values of the quark constituent masses, these energies are also smooth functions of $\mu$.

We next observe that $E(i j)$ and $E(i j k)$, considered as functions of $\mu(i j)$ and $\mu(i j k)$ respectively, depend most sensitively on the mass of the lightest quark. We therefore tend to have a small change in $\mu(i j)$ and $\mu(i j k)$ if we confine ourselves to the case in which initially all the quarks in a hadron are $u$ or $d$ quarks (denoted by $n$ ), and only one of the quarks (say the $j$ quark) is allowed to change from an $n$ quark to a heavier $s, c$, or $b$ quark (denoted by $h$ ). Because the $E(i j)$ and $E(i j k)$ are smooth functions of $\mu(i j)$ and $\mu(i j k)$ respectively, small changes in the reduced masses will lead to small changes in the interaction energies. Consequently, if $\mu(453)$ is close to $\mu(123)$ and if $\mu(45)$ is close to $\mu(12)$, the value of $\delta(12345)$, given in Eq. (4), will be small and we shall neglect it. We insure this to be the case by confining ourselves to hadrons containing at most one quark which is not a light $n$ quark; specifically, we assume

$$
\delta(n n n n q)=0,
$$

where $q$ is any quark. Then, from Eq. (5) we have the sum rule

$$
M_{B}(n n n)-M_{M}(n n)=M_{B}(n h n)-M_{M}(n h) .
$$

We now consider the effect of spin-dependent forces. We let the spin-dependent colormagnetic interaction be a perturbation of the form [1]

$$
V_{c m}=-\sum_{i<j} \sigma_{i} \cdot \sigma_{j} \lambda_{i} \cdot \lambda_{j} f\left(r_{i j}\right) /\left(m_{i} m_{j}\right),
$$

where the $\sigma_{i}$ are Pauli spin matrices, the $\lambda_{i}$ are Gell-Mann matrices, and $f\left(r_{i j}\right)$ is a positive-definite operator which depends on the separation $r_{i j}$ between quarks $i$ and $j$. We do not need to specify the functional form of $f\left(r_{i j}\right)$. Then it is straightforward to take hadron spin averages [3]. We obtain three sum rules from Eq. (8):

$$
(N+\Delta) / 2-(3 \rho+\pi) / 4=\left(2 \Sigma^{*}+\Sigma+\Lambda\right) / 4-\left(3 K^{*}+K\right) / 4
$$

if $h=s$,

$$
(N+\Delta) / 2-(3 \rho+\pi) / 4=\left(2 \Sigma_{c}^{*}+\Sigma_{c}+\Lambda_{c}\right) / 4-\left(3 D^{*}+D\right) / 4
$$

if $h=c$, and

$$
(N+\Delta) / 2-(3 \rho+\pi) / 4=\left(2 \Sigma_{b}^{*}+\Sigma_{b}+\Lambda_{b}\right) / 4-\left(3 B^{*}+B\right) / 4
$$

if $h=b$, where the symbol for a hadron denotes its mass. 
We can test the first of these sum rules with data from main tables of the Particle Data Group [4]. We obtain that the left hand side of Eq. (10) is $474 \mathrm{Mev}$, while the right hand side is $473 \mathrm{MeV}$, giving excellent agreement with experiment. Turning to Eq. (11), we find that all masses except that of the $\Sigma_{c}^{*}$ are well known, but the $\Sigma_{c}^{*}$ is given as a poorly established state of mass $2530 \pm 10$ $\mathrm{MeV}$. We therefore use Eq. (11) to predict the mass of the $\Sigma_{c}^{*}$, obtaining

$$
\Sigma_{c}^{*}=2523 \mathrm{MeV}
$$

The agreement between our prediction and the value given by the Particle Data Group is sufficiently close to lend theoretical support to the experimental evidence [5] that the $\Sigma_{c}^{*}$ baryon has indeed been found. At the present time, the sum rule given in Eq. (12) cannot be tested because the mass of the $\Lambda_{b}$ is known only with an error of $50 \mathrm{MeV}$ and the $\Sigma_{b}$ and $\Sigma_{b}^{*}$ have not yet been observed. However, we hope that Eq. (12) can be tested in the not-too-distant future.

In conclusion, we have obtained three sum rules relating meson and baryon masses. Two of these formulas agree with existing experimental data, and the third should be testable by future experiments. Although we have obtained our results within the framework of the constituent quark

model and the Hamiltonian formalism, we have not had to give explicitly either the Hamiltonian or the quark masses.

This work was supported in part by the U. S. Department of Energy and in part by the U. S. National Science Foundation.

\section{References}

[1] R. Roncaglia, A. Dzierba, D.B. Lichtenberg, and E. Predazzi, Phys. Rev. D 51, 1248 (1995).

[2] R. Roncaglia, D.B. Lichtenberg, and E. Predazzi, Indiana Univ. report No. IUHET 293 (1995), Phys. Rev. D (to be published).

[3] M. Anselmino, D. B. Lichtenberg, and E. Predazzi, Z. Phys. C 48, 605 (1990).

[4] Particle Data Group: L. Montanet et al., Phys. Rev. D 50, 1173 (1994).

[5] V. V. Ammosov et al., Pis'ma Zh. Eksp. Teor. Fiz. 58, 241 (1993) [JETP Lett. 58, 247 (1993)]. 